\title{
Fractional Quantum Mechanics and Lévy Path Integrals
}

\author{
Nikolai Laskin* \\ Isotrace Laboratory, University of Toronto \\ 60 St. George Street, Toronto, ON M5S 1A7 \\ Canada
}

\begin{abstract}
The fractional quantum and statistical mechanics have been developed via new path integrals approach.

PACS number(s): 03.65.-w, 05.30.-d, 05.40. Fb, 03.65. Db
\end{abstract}

From a functional integration point of view the quantum mechanics in Feynman's path integrals treatment [1], [2] is the theory of functionals on measure generated by the Brownian motion-Wiener stochastic process [3], [四]. The Wiener process is the Markov, Gaussian or normal stochastic process the statistical description of which is given by well-known diffusion equation [曰].

The natural generalization of the Brownian motion is the Lévy flights (or Lévy motion). The mathematical foundation of this generalization is the theory of stable probability distributions developed by Lévy [5]. The most fundamental property of the Lévy distributions is the stability under addition, following from the generalized central limit theorem valid for Lévy distributions. So, from the probability theory point of view the stable probability law is the generalization of well-known Gaussian law.

Lévy flights is widely used to model a variety processes such as anomalous diffusion [6], turbulence [0], chaotic dynamics [8], plasma physics [9],

*E-mail: nlaskin@rocketmail.com 
financial dynamics [10], biology and physiology [11]. The most recent studies are related to fractional Lévy motion [12] which is the generalization of the fractional Brownian motion 6].

This paper deals with new path integrals over measures induced by the stochastic process of Lévy flights. I have generalized the Feynman and Wiener path integrals and developed the new fractional quantum mechanics (fQM) and fractional statistical mechanics (fSM). The fQM and fSM include the Feynman quantum and statistical mechanics in similar way as the Lévy process (Lévy flights) generalizes the Wiener process (Brownian motion).

In this paper I have developed the foundations of fQM and fSM in their functional formulations. As the next step we should think about the concrete fQM problems such as, for example, fractional harmonic oscillator, fractional hydrogen atom, fractional scattering theory, relativistic fractional quantum mechanics, fractional quantum gauge field theory etc.

1. The Feynman quantum mechanics deals with Feynman's quantum mechanical functionals $K_{F}\left(x_{b} t_{b} \mid x_{a} t_{a}\right)$ [1]

$$
K_{F}\left(x_{b} t_{b} \mid x_{a} t_{a}\right)=\int_{x\left(t_{a}\right)=x_{a}}^{x\left(t_{b}\right)=x_{b}} \mathcal{D}_{\text {Feynman }} x(\tau) \cdot \exp \left\{\frac{i}{\hbar} \int_{t_{a}}^{t_{b}} d \tau V(x(\tau))\right\},
$$

where $V(x(\tau))$ is the potential energy as a functional of a particle path $x(\tau)$ and the Feynman functional measure is defined as

$$
\begin{gathered}
\int_{x\left(t_{a}\right)=x_{a}}^{x\left(t_{b}\right)=x_{b}} \mathcal{D}_{\text {Feynman }} x(\tau) \ldots=\lim _{N \rightarrow \infty} \int d x_{1} \ldots d x_{N-1}\left(\frac{2 \pi i \hbar \varepsilon}{m}\right)^{-N / 2} \times \\
\times \prod_{j=1}^{N} \exp \left\{\frac{i m}{2 \hbar \varepsilon}\left(x_{j}-x_{j-1}\right)^{2}\right\} \ldots,
\end{gathered}
$$

here $m$ is the mass of the quantum mechanical particle, $\hbar$ is the Planck's constant, $x_{0}=x_{a}, x_{N}=x_{b}$ and $\varepsilon=\left(t_{b}-t_{a}\right) / N$. 
I propose the fractional quantum mechanics based on the new path integral

$$
K_{L}\left(x_{b} t_{b} \mid x_{a} t_{a}\right)=\int_{x\left(t_{a}\right)=x_{a}}^{x\left(t_{b}\right)=x_{b}} \mathcal{D}_{\text {Laskin }} x(\tau) \cdot \exp \left\{\frac{i}{\hbar} \int_{t_{a}}^{t_{b}} d \tau V(x(\tau))\right\},
$$

where $V(x(\tau))$ is the potential energy as a functional of the Lévy particle path and the Laskin path integral measure is defined as

$$
\begin{gathered}
\int_{x\left(t_{a}\right)=x_{a}}^{x\left(t_{b}\right)=x_{b}} \mathcal{D}_{\text {Laskin }} x(\tau) \ldots= \\
=\lim _{N \rightarrow \infty} \int d x_{1} \ldots d x_{N-1}\left(\frac{i D_{\alpha} \varepsilon}{\hbar}\right)^{-N / \alpha} \cdot \prod_{j=1}^{N} L_{\alpha}\left\{\left(\frac{\hbar}{i D_{\alpha} \varepsilon}\right)^{1 / \alpha}\left|x_{j}-x_{j-1}\right|\right\} \ldots
\end{gathered}
$$

where $D_{\alpha}$ is the generalized "diffusion coefficient", $\hbar$ denotes the Planck's constant, $x_{0}=x_{a}, x_{N}=x_{b}, \varepsilon=\left(t_{b}-t_{a}\right) / N$, and the Lévy function $L_{\alpha}$ is expressed in terms of Fox's $H$ functions [13], [14, [15]

$$
\begin{gathered}
\left(\frac{D_{\alpha} t}{\hbar}\right)^{-1 / \alpha} L_{\alpha}\left\{\left(\frac{\hbar}{D_{\alpha} t}\right)^{1 / \alpha}|x|\right\}= \\
=\frac{\pi}{\alpha|x|} H_{2,2}^{1,1}\left[\left(\frac{\hbar}{D_{\alpha} t}\right)^{1 / \alpha}|x| \mid \begin{array}{c}
(1,1 / \alpha),(1,1 / 2) \\
(1,1),(1,1 / 2)
\end{array}\right],
\end{gathered}
$$

here $\alpha$ is the Lévy index, $0<\alpha \leq 2$.

The Eqs (3)-(5) define the fractional quantum mechanics via Laskin path integral.

The fractional free particle propagator $K_{L}^{(0)}\left(x_{b} t_{b} \mid x_{a} t_{a}\right)$ has the form

$$
K_{L}^{(0)}\left(x_{b} t_{b} \mid x_{a} t_{a}\right)=\int_{x\left(t_{a}\right)=x_{a}}^{x\left(t_{b}\right)=x_{b}} \mathcal{D}_{\text {Laskin }} x(\tau) \cdot 1=
$$




$$
=\left(\frac{i D_{\alpha}\left(t_{b}-t_{a}\right)}{\hbar}\right)^{-1 / \alpha} L_{\alpha}\left\{\left(\frac{\hbar}{i D_{\alpha}\left(t_{b}-t_{a}\right)}\right)^{1 / \alpha}\left|x_{b}-x_{a}\right|\right\} .
$$

It is known that at $\alpha=2$ the Lévy distribution is transformed to Gaussian and the Lévy flights process is transformed to the process of the Brownian motion. The Eq.(6) in accordance with definition given by the Eq.(5) and the properties of the Fox's function $H_{2,2}^{1,1}$ at $\alpha=2$ (see, [14, [15]) is transformed to Feynman free particle propagator 11

$$
K_{F}^{(0)}\left(x_{b} t_{b} \mid x_{a} t_{a}\right)=\left(\frac{2 \pi i \hbar\left(t_{b}-t_{a}\right)}{m}\right)^{-1 / 2} \cdot \exp \left\{\frac{i m\left(x_{b}-x_{a}\right)^{2}}{2 \hbar\left(t_{b}-t_{a}\right)}\right\} .
$$

In terms of Fourier integral (momentum representation) the propagator $K_{L}^{(0)}\left(x_{b} t_{b} \mid x_{a} t_{a}\right)$ is written as follows

$$
K_{L}^{(0)}\left(x_{b} t_{b} \mid x_{a} t_{a}\right)=\frac{1}{2 \pi \hbar} \int d p \cdot \exp \left\{i \frac{p\left(x_{b}-x_{a}\right)}{\hbar}-i \frac{D_{\alpha}\left(t_{b}-t_{a}\right)|p|^{\alpha}}{\hbar}\right\} .
$$

We see that the energy $E_{p}$ of the fractional quantum mechanical particle with the momentum $p$ is given by

$$
E_{p}=D_{\alpha}|p|^{\alpha} .
$$

The comparison of the Eq.(17) and the Eq.(8) allows to conclude that at $\alpha=2$ we should put $D_{2}=1 / 2 \mathrm{~m}$. Then the Eq.(9) is transformed to $E_{p}=p^{2} / 2 m$.

Using the Eq.(9) we can define the Laskin functional measure in the momentum representation by the following way

$$
\begin{gathered}
\int_{x\left(t_{a}\right)=x_{a}}^{x\left(t_{b}\right)=x_{b}} \mathcal{D}_{\text {Laskin }} x(\tau) \ldots= \\
=\lim _{N \rightarrow \infty} \int d x_{1} \ldots d x_{N-1} \frac{1}{(2 \pi \hbar)^{N}} \int d p_{1} \ldots d p_{N} \cdot \exp \left\{i \frac{p_{1}\left(x_{1}-x_{a}\right)}{\hbar}-i \frac{D_{\alpha} \varepsilon\left|p_{1}\right|^{\alpha}}{\hbar}\right\} \times \ldots
\end{gathered}
$$




$$
\times \exp \left\{i \frac{p_{N}\left(x_{b}-x_{N-1}\right)}{\hbar}-i \frac{D_{\alpha} \varepsilon\left|p_{N}\right|^{\alpha}}{\hbar}\right\} \cdots
$$

here $\varepsilon=\left(t_{b}-t_{a}\right) / N$.

Taking into account the Eq.(8) it is easy to check on the consistency condition

$$
K_{L}^{(0)}\left(x_{b} t_{b} \mid x_{a} t_{a}\right)=\int d x^{\prime} K_{L}^{(0)}\left(x_{b} t_{b} \mid x^{\prime} t^{\prime}\right) \cdot K_{L}^{(0)}\left(x^{\prime} t^{\prime} \mid x_{a} t_{a}\right) .
$$

The fractional free particle propagator is governed by the fractional Schrödinger equation

$$
i \hbar \frac{\partial}{\partial t_{b}} K_{L}^{(0)}\left(x_{b} t_{b} \mid x_{a} t_{a}\right)=-D_{\alpha}\left(\hbar \nabla_{b}\right)^{\alpha} K_{L}^{(0)}\left(x_{b} t_{b} \mid x_{a} t_{a}\right),
$$

where the Riesz quantum fractional derivative $(\hbar \nabla)^{\alpha}$ is defined through its Fourier transform (see, for example, [16])

$$
(\hbar \nabla)^{\alpha} \ldots=-\frac{1}{2 \pi \hbar} \int d p e^{i \frac{p x}{\hbar}}|p|^{\alpha} \ldots
$$

The propagator $K_{L}\left(x_{b} t_{b} \mid x_{a} t_{a}\right)$ describes the evolution of the fractional quantum-mechanical system

$$
\psi_{f}\left(x_{b}, t_{b}\right)=\int d x_{a} K_{L}\left(x_{b} t_{b} \mid x_{a} t_{a}\right) \cdot \psi_{i}\left(x_{a}, t_{a}\right)
$$

where $\psi_{i}\left(x_{a}, t_{a}\right)$ is the fractional wave function of initial (at the $t=t_{a}$ ) state and $\psi_{f}\left(x_{b}, t_{b}\right)$ is the fractional wave function of final (at the $t=t_{b}$ ) state.

Taking into account the Eqs.(3), (14) and the definition (13) we can derive the fractional Schrödinger equation for the wave function of quantum mechanical system

$$
i \hbar \frac{\partial \psi}{\partial t}=-D_{\alpha}(\hbar \nabla)^{\alpha} \psi+V(x) \psi
$$

It is easy to see that the fractional (or Levy) free particle wave function has the form 


$$
\psi(x, t)=\frac{1}{2 \pi \hbar} \int d p \cdot \exp \left\{i \frac{p x}{\hbar}-i \frac{D_{\alpha} t|p|^{\alpha}}{\hbar}\right\} .
$$

Thus, the Eqs.(3), (41) and (14), (15) are the basic equations of new fQM. They should be analyzed for the particular fractional quantum systems.

2. In order to develop the fractional statistical mechanics let us go in the previous consideration from imaginary time to "inverse temperature" $\beta=1 / k_{B} T$, where $k_{B}$ is the Boltzmann's constant and $T$ is the temperature, $i t \rightarrow \beta$. In the framework of traditional functional approach to the statistical mechanics we deal with functionals over the Wiener measure [1], [4], [17]

$$
\rho_{W}\left(x, \beta \mid x_{0}\right)=\int_{x(0)=x_{0}}^{x(\beta)=x} \mathcal{D}_{\text {Wiener }} x(u) \cdot \exp \left\{-\int_{0}^{\beta} d u V(x(u))\right\},
$$

where $\rho_{W}\left(x, \beta \mid x_{0}\right)$ is the density matrix of the statistical system in the external field $V(x)$ and the Wiener measure is given by

$$
\begin{gathered}
\int_{x(0)=x_{0}}^{x(\beta)=x} \mathcal{D}_{\text {Wiener }} x(u) \ldots=\lim _{N \rightarrow \infty} \int d x_{1} \ldots d x_{N-1}\left(\frac{2 \pi \hbar^{2} \varsigma}{m}\right)^{-N / 2} \times \\
\times \prod_{j=1}^{N} \exp \left\{-\frac{m}{2 \hbar^{2} \varsigma}\left(x_{j}-x_{j-1}\right)^{2}\right\} \ldots,
\end{gathered}
$$

here $\varsigma=\beta / N$ and $x_{N}=x$.

The fSM deals with Lévy or fractional density matrix $\rho_{L}\left(x, \beta \mid x_{0}\right)$ which is given by

$$
\rho_{L}\left(x, \beta \mid x_{0}\right)=\int_{x(0)=x_{0}}^{x(\beta)=x} \mathcal{D}_{\text {Lévy }} x(u) \cdot \exp \left\{-\int_{0}^{\beta} d u V(x(u))\right\},
$$

where the Lévy functional measure is defined as follows 


$$
\begin{gathered}
\int_{x(0)=x_{0}}^{x(\beta)=x} \mathcal{D}_{\text {Lévy }} x(u) \ldots=\lim _{N \rightarrow \infty} \int d x_{1} \ldots d x_{N-1}\left(D_{\alpha} \varsigma\right)^{-N / \alpha} \times \\
\prod_{j=1}^{N} L_{\alpha}\left\{\frac{\left|x_{j}-x_{j-1}\right|}{\left(D_{\alpha} \varsigma\right)^{1 / \alpha}}\right\} \ldots,
\end{gathered}
$$

here $\varsigma=\beta / N, x_{N}=x$ and the Lévy function $L_{\alpha}$ is given by the Eq.(5).

It was shown by Lévy [18] (and later by Doob [19]) that the Eq.(20) defines the functional measure in the space $\mathfrak{D}$ of left (or right) continuous functions having only discontinuities of the first kind.

In Fourier space the fractional density matrix $\rho_{L}^{(0)}\left(x, \beta \mid x_{0}\right)$ of a free particle $(V=0)$ has the form

$$
\rho_{L}^{(0)}\left(x, \beta \mid x_{0}\right)=\frac{1}{2 \pi \hbar} \int d p \exp \left\{i \frac{p\left(x-x_{0}\right)}{\hbar}-\beta D_{\alpha}|p|^{\alpha}\right\} .
$$

When $\alpha=2$ and $D_{2}=1 / 2 m$ the Eq.(21) gives the well-known density matrix for one-dimensional free particle [17]

$$
\rho_{W}^{(0)}\left(x, \beta \mid x_{0}\right)=\left(\frac{m}{2 \pi \hbar^{2} \beta}\right)^{1 / 2} \exp \left\{-\frac{m}{2 \hbar^{2} \beta}\left(x-x_{0}\right)^{2}\right\} .
$$

The density matrix $\rho_{L}\left(x, \beta \mid x_{0}\right)$ obeys the fractional differential equation

$$
-\frac{\partial \rho_{L}\left(x, \beta \mid x_{0}\right)}{\partial \beta}=-D_{\alpha}\left(\hbar \nabla_{x}\right)^{\alpha} \rho_{L}\left(x, \beta \mid x_{0}\right)+V(x) \rho_{L}\left(x, \beta \mid x_{0}\right) .
$$

The Eqs. (19) - (21) and (23) are the basic equations of new fSM in its functional formulation.

3. I have developed the new path integral approach to fQM and fSM. My approach is based on the new functional measures generated by the stochastic process of the Lévy flights. The Laskin and Lévy path integrals are the generalization of the Feynman and Wiener path integrals respectively.

It will be interesting to find the physical systems and objects the quantum dynamics (statistical mechanics) of which is governed by the developed fQM (fSM). 


\section{References}

[1] R. P. Feynman and A.R. Hibbs, Quantum Mechanics and Path Integrals (McGraw-Hill, New York, 1965).

[2] C. Grosche and F. Steiner, Handbook of Feynman Path Integrals (Springer-Verlag, Berlin Heidelberg, 1998).

[3] N. Wiener, Proc. Lond. Math. Soc. 22, 454 (1924).

[4] M. Kac, Probability and Related Topics in Physical Sciences (Interscience, New York, 1959), Chap. IV.

[5] P. Lévy, Théorie de l'Addition des Variables Aléatoires (GauthierVillars, Paris, 1937).

[6] B.B. Mandelbrot and J.W. van Ness, SIAM (Soc. Ind. Appl. Math.) Rev. 10, 422 (1968).

[7] J. Klafter, A.Blumen and M.F. Shlesinger, Phys. Rev. A35, 3081 (1987).

[8] G.M. Zaslavsky, Physica D76, 110 (1994).

[9] G. Zimbardo, P. Veltri, G. Basile and S. Principato, Phys. Plasmas 2 (7), 2653 (1995).

[10] R.N. Mantega and H.E. Stanley, Nature, 376 , 46 (1995).

[11] B.J. West and W. Deering, Phys. Rep. 246, 1 (1994).

[12] A. V. Chechkin, V. Yu. Gonchar, cond-mat/9903400.

[13] C. Fox, Trans. Am. Math. Soc. 98, 395 (1961).

[14] A.M. Mathai and R.K. Saxena, The H-function with Applications in Statistics and Other Disciplines (Wiley Eastern, New Delhi, 1978).

[15] B.J. West and P. Grigolini, Phys. Rev. E55, 99 (1997).

[16] K.B. Oldham and J. Spanier, The Fractional Calculus (Academic Press, New York, 1974). 
[17] R.P. Feynman, Statistical Mechanics (Benjamin. Reading, Mass. 1972).

[18] P. Lévy, Ann. Pisa, 3, 337 (1934).

[19] J.L. Doob, Stochastic processes (Wiley, New York 1953). 